\title{
ERGODIC AND MIXING PROPERTIES OF \\ CHEBYSHEV POLYNOMIALS
}

R. L. ADLER AND T. J. RIVLIN

A sequence $\left\{T_{n}: n=1,2, \cdots\right\}$ of, not necessarily invertible, measurable transformations on a finite measure space, $(X, \mathfrak{B}, \mu)$, preserving the measure $\mu$ in the sense that $\mu\left(T_{n}^{-1} B\right)=\mu(B)$ for $B \in \mathfrak{B}$, is called strongly mixing if

$$
\lim _{n \rightarrow \infty} \mu\left(T_{n}^{-1} A \cap B\right)=\frac{\mu(A) \mu(B)}{\mu(X)}
$$

for $A, B \in \mathfrak{B}$.

Let $X$ denote the interval $[-1,1], \mathfrak{B}$ the family of Borel subsets of $X, \lambda$ Lebesgue measure and $\mu$ the equivalent measure defined by

$$
\mu(B)=\frac{2}{\pi} \int_{B} \frac{\lambda(d x)}{\sqrt{ }\left(1-x^{2}\right)}, \quad B \in \mathfrak{B} .
$$

The Chebyshev polynomial of degree $n$ is defined as $T_{n}(x)=\cos n \theta$, where $x=\cos \theta, 0 \leqq \theta \leqq \pi$. If we put $T_{0}(x)=1 / \sqrt{ } 2$ then it is well known that the sequence $\left\{T_{k}(x): k=0,1,2, \cdots\right\}$ is a complete orthonormal set (c.o.n.s.) in $L^{2}(X, \mathfrak{B}, \mu)$. Let $T_{n}$ be the transformation defined by $T_{n}(x)$, then $T_{n}$ is a measurable almost everywhere $n$ to one mapping of $X$ onto itself satisfying $T_{n}\left(T_{m}\right)=T_{n m}$ for $n, m=1,2, \cdots$, i.e. $\left\{T_{n}: n=1,2, \cdots\right\}$ is a semi-group under composition. (The semi-group property is a simple consequence of the definition of the Chebyshev polynomials.)

Lemma. $T_{n}$ preserves the measure $\mu$ for $n=1,2, \cdots$.

Proof. Consider the measure space $\left(X^{\prime}, \mathfrak{B}^{\prime}, \lambda^{\prime}\right)$ where $X^{\prime}$ is the interval $[0, \pi], \mathfrak{B}^{\prime}$ the Borel field of subsets of $X^{\prime}$ and $\lambda^{\prime}$ Lebesgue measure on $\mathfrak{B}^{\prime}$. Let $R$ denote the one to one measurable mapping of $X$ onto $X^{\prime}$ determined by $x \rightarrow x^{\prime}=\operatorname{arc} \cos x$, and define $S_{n}$ by $S_{n}$ $=R T_{n} R^{-1}$. If $k \pi / n \leqq x^{\prime} \leqq(k+1) \pi / n, k=0,1, \cdots, n-1$, we observe that $S_{n}\left(x^{\prime}\right)=n x^{\prime}-k \pi, k$ even; $S_{n}\left(x^{\prime}\right)=-n x^{\prime}+(k+1) \pi, k$ odd, and thus $S_{n}$ preserves Lebesgue measure for $n=1,2, \cdots$. A standard change of variables now reveals that

$$
\int_{a}^{b} \frac{d x}{\sqrt{ }\left(1-x^{2}\right)}=\int_{R(a)}^{R(b)} d x^{\prime}, \quad-1 \leqq a<b \leqq 1,
$$

Received by the editors March 29, 1963 and, in revised form, May 3, 1963. 
which implies that $\mu(A)=\lambda^{\prime}(R A)$ for $A \in \mathfrak{B}$. Therefore $\mu\left(T_{n}^{-1} A\right)$ $=\lambda^{\prime}\left(R T_{n}^{-1} A\right)=\lambda^{\prime}\left(R T_{n}^{-1} R^{-1} R A\right)=\lambda^{\prime}\left(S_{n}^{-1} R A\right)=\lambda^{\prime}(R A)=\mu(A)$ for $A \in \mathfrak{B}$ and $n=1,2, \cdots$.

THEOREM. The sequence of transformations $\left\{T_{n}: n=1,2, \cdots\right\}$ is strongly mixing with respect to the measure $\mu$.

Proof. To establish (1) it suffices to prove

$$
\lim _{n \rightarrow \infty} \int_{X} f\left(T_{n} x\right) g(x) d \mu(x)=\frac{1}{\mu(X)} \int_{X} f d \mu(x) \cdot \int_{X} g d \mu(x)
$$

for $f, g \in L^{2}(X, \mathfrak{B}, \mu)$, since if $f$ is the characteristic function of $A$ and $g$ is the characteristic function of $B,(2)$ becomes (1). It is sufficient to establish (2) for $f, g$ restricted to $\left\{T_{k}: k=0,1,2, \cdots\right\}$. Having done this the fact that $\left\{T_{k}: k=0,1,2, \cdots\right\}$ is a c.o.n.s. allows us to prove (2) by a standard approximation argument. In view of the orthogonality and semi-group properties of the Chebyshev polynomials (2) is easy to verify for $f$ and $g$ restricted to $\left\{T_{k}: k=0,1,2, \cdots\right\}$.

An application of the theorem. Let $A_{n}\left(y_{2}\right)$ be the area under the graph of $y=T_{n}(x)$ contained between the lines $x=-1, x=1, y=-1$ and $y=y_{2}$ where $-1<y_{2} \leqq 1$. We wish to establish the existence and determine the value of

$$
A\left(y_{2}\right)=\lim _{n \rightarrow \infty} A_{n}\left(y_{2}\right) .
$$

We proceed as follows: Let $g(x)=\sqrt{ }\left(1-x^{2}\right)$ and $f(x)=y_{2}, y_{2} \leqq x$; $f(x)=x,-1 \leqq x \leqq y_{2}$. Then

$$
\begin{aligned}
A\left(y_{2}\right) & =2+\lim _{n \rightarrow \infty} \int_{-1}^{1} f\left(T_{n}(x)\right) d x \\
& =2+\lim _{n \rightarrow \infty} \frac{\pi}{2} \int_{X} f\left(T_{n}(x)\right) g(x) d \mu=2+\frac{\pi}{2 \mu(X)} \int_{X} f d \mu \cdot \int_{X} g d \mu \\
& =2+\frac{2}{\pi} \int_{-1}^{1} f(x)\left(1-x^{2}\right)^{-1 / 2} d x \\
& =2+\frac{2}{\pi}\left[\int_{-1}^{y_{2}} x\left(1-x^{2}\right)^{-1 / 2} d x+\int_{y_{2}}^{1} y_{2}\left(1-x^{2}\right)^{-1 / 2} d x\right] .
\end{aligned}
$$

Thus, performing these integrations we obtain

$$
A\left(y_{2}\right)=2+y_{2}-\frac{2}{\pi}\left[\left(1-y_{2}^{2}\right)^{1 / 2}+y_{2} \arcsin y_{2}\right] .
$$


Furthermore, the limit as $n \rightarrow \infty$ of the area under $y=T_{n}(x)$ and between the lines $x=x_{1}, x=x_{2}, y=-1$ and $y=y_{2}$, where $-1 \leqq x_{1}<x_{2} \leqq 1$, $-1<y_{2} \leqq 1$ is $\left(x_{2}-x_{1}\right) A\left(y_{2}\right) / 2$. This is readily obtained by taking for $g(x)$ the product of the characteristic function of $\left[x_{1}, x_{2}\right]$ and $\left(1-x^{2}\right)^{1 / 2}$. Therefore, the limit as $n \rightarrow \infty$ of the area under $y=T_{n}(x)$ and in the region bounded by the vertical lines $x=x_{1}, x=x_{2}$ and the continuous curves $y=y_{1}(x), y=y_{2}(x)$, where $-1 \leqq x_{1}<x_{2} \leqq 1$, $-1 \leqq y_{1}(x)<y_{2}(x) \leqq 1$ for $x$ in $\left[x_{1}, x_{2}\right]$ is

$$
\frac{1}{2} \int_{x}^{x_{2}}\left[A\left(y_{2}(x)\right)-A\left(y_{1}(x)\right)\right] d x .
$$

REMARKs. It follows from the Theorem (in view of the semi-group property) that for $n>1$ any particular $T_{n}$ is strongly mixing in the sense that

$$
\lim _{k \rightarrow \infty} \mu\left(T_{n}^{-k} A \cap B\right)=\frac{\mu(A) \mu(B)}{\mu(X)} .
$$

Moreover, this implies that the $T_{n}, n>1$, are ergodic transformations, i.e., if $T_{n}^{-1} A=A, A \in \mathfrak{B}$, then either $\mu(A)=0$ or $\mu(A)=\mu(X)$.

It is perhaps of interest to compare the transformations $S_{n}$ to the more familiar transformations $Q_{n}: x \rightarrow n x(\bmod 1)$, defined on $[0,1]$, which also form a strongly mixing sequence. The accompanying diagrams illustrate the case $n=3$.

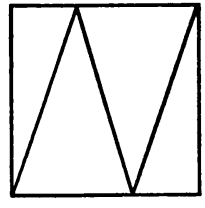

$S_{3}$

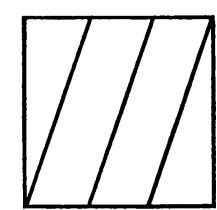

$Q_{3}$

The strong mixing of $\left\{T_{n}: n=1,2, \cdots\right\}$ also can be deduced from the strong mixing of $\left\{Q_{n}: n=1,2, \cdots\right\}$ without requiring the two semi-groups of measure preserving transformations to be spatially isomorphic in the sense that there exists an isomorphism $R$ between $(X, \mathfrak{B}, \mu)$ and $\left(X^{\prime}, \mathfrak{B}^{\prime}, \lambda^{\prime}\right)$ such that $R T_{n} R^{-1}=Q_{n}$.

The authors are grateful to Professor S. Kakutani for helpful advice about the results presented here.

International Business Machines Corporation, Yorktown Heights 